\title{
CARACTERIZACIÓN DE LOS SÍNTOMAS VISUALES DE DEFICIENCIAS NUTRICIONALES DE PLÁNTULAS DEL ROBLE ANDINO EN INVERNADERO
}

\author{
Yira Lucia Sepúlveda*, María Claudia Díez, ${ }^{* *}$ Nelson Walter Osorio ${ }^{1 * * *}$, \\ Flavio Humberto Moreno**, Juan Diego León ${ }^{* *}$
}

Palabras clave: Fertilización, nutrición forestal, Quercus humboldtii, roble, vivero.
Keywords: Fertilization, forest nutrition, Quercus humboldtii, oak, nursery.

Recibido: 17/09/13

\section{RESUMEN}

La identificación de síntomas visuales de deficiencias es un método muy utilizado en el diagnóstico de problemas nutricionales en la producción agrícola y forestal. El objetivo de este trabajo fue caracterizar los síntomas visuales de deficiencias de N, P, K, Ca, Mg, S, y B, en plántulas de roble andino Quercus humboldtii Bonpl., en condición de vivero. Las plántulas se sembraron en bolsas de polietileno, en cuyos sustratos se aplicaron los tratamientos de fertilización de acuerdo con el método de Jenny. Luego de 4 meses de desarrollo se fotografiaron las plántulas y hojas en las que se observaron los síntomas de deficiencias nutricionales y se determinó el contenido de nutrientes foliares. Se detectaron síntomas de deficiencia de todos los nutrientes estudiados, excepto de B. Las plántulas deficientes en cada nutriente presentaron menor concentración de éste elemento en los tejidos foliares. La dosis aplicada de $\mathrm{P}$ al parecer no fue suficiente para tener una disponibilidad adecuada del elemento y generar un óptimo desarrollo de las plántulas de $Q$. humboldtii con tratamiento completo de fertilización.

\footnotetext{
1 Autor para correspondencia. Correo electrónico: nwosorio@unal.edu.co

Maestría en Bosques y Conservación. Universidad Nacional de Colombia, Medellín, Colombia.
}

Aceptado: 03/02/14

\begin{abstract}
Characterization of visual symptoms of nutritional deficiencies in oak seedlings. The identification of visual symptoms of deficiencies is a widely used method in the diagnosis of nutritional problems in agricultural and forestry production. The aim of this study was to characterize visual symptoms of deficiencies of $\mathrm{N}, \mathrm{P}$, $\mathrm{K}, \mathrm{Ca}, \mathrm{Mg}, \mathrm{S}$ and $\mathrm{B}$, in seedlings of the andean oak, Quercus humboldtii Bonpl., under nursery conditions. Seedlings were planted in polythene bags, where fertility treatments were applied according to the method of Jenny. After 4 months of development, we photographed seedlings and leaves with symptoms of nutritional deficiencies and determined leaf nutrient content. Deficiency symptoms were detected for all nutrients studied, except B. Seedlings deficient in each nutrient had lower concentration of this element in leaf tissues. The applied dose of $\mathrm{P}$ was apparently not enough to have an adequate availability of this nutrient and to generate an optimal development of seedlings of $Q$. humboldtii under full fertilization.
\end{abstract}

\footnotetext{
** Departamento de Ciencias Forestales. Universidad Nacional de Colombia, Medellín, Colombia.

*** Escuela de Biociencias. Universidad Nacional de Colombia, Medellín, Colombia.
} 


\section{INTRODUCCIÓN}

Los nutrientes cumplen un papel esencial y específico en la fisiología vegetal; cuando uno de estos elementos no se encuentra en las cantidades adecuadas su deficiencia en los tejidos promueve cambios en el metabolismo de la planta (Carpanezzi et ál. 1999). Los síntomas de deficiencia nutricional son más o menos característicos de cada nutriente y dependen de la gravedad de la deficiencia (Santi et ál. 2006). El grado de movilidad que presentan los elementos en las plantas determina la localización de los síntomas de su deficiencia. Algunos nutrientes como nitrógeno $(\mathrm{N})$, fósforo $(\mathrm{P})$ y potasio $(\mathrm{K})$ se consideran muy móviles, por lo cual las plántulas deficientes en estos elementos inicialmente presentan síntomas visuales en las hojas más viejas. Por el contrario, las plántulas deficientes en calcio $(\mathrm{Ca})$, azufre (S) y boro (B) presentan síntomas en las hojas jóvenes ya que estos nutrientes son poco móviles.

La información sobre el diagnóstico de los síntomas visuales de deficiencias nutricionales tiene gran utilidad en el manejo de viveros forestales, pues permite reconocer rápidamente problemas en la nutrición de las plántulas que pueden afectar el éxito del establecimiento (Wallau et ál. 2008, Sarcinelli et ál. 2004). Esta técnica también es útil en el campo ya que representa una herramienta auxiliar para establecer la necesidad de aplicar fertilizantes o enmiendas (Wallau et ál. 2008). La asociación de síntomas de deficiencias nutricionales con el contenido de nutrientes en los tejidos foliares, puede servir para determinar concentraciones adecuadas y deficientes asociadas con problemas de desarrollo en las plantas. En Colombia varias entidades han realizado investigaciones sobre síntomas visuales de deficiencias nutricionales en cultivos de importancia comercial: el CIAT (en leguminosas y gramíneas), el ICA (en cacao), CENICAFE (en café), CENIBANANO (en banano), entre otros. Esta técnica ha sido aplicada en pocas especies forestales (Carpanezzi et ál. 1999). Sin embargo, se conocen algunos estudios con especies nativas de importancia comercial en el hemisferio sur tales como caobo (Swietenia macrophylla) (Wallau et ál. 2008), acacia (Acacia holosericea) (Sarcinelli et ál. 2004) y jatobá (Hymenaea courbaril) (Duboc 1994).

Debido al alto grado de amenaza y a los problemas de conservación de Quercus humboldtii, una especie endémica del norte de los Andes, es urgente su establecimiento asistido en programas de restauración de ecosistemas y en plantaciones comerciales para disminuir la presión sobre el ecosistema natural. Para ello se hace necesario el estudio de aspectos relacionados con la nutrición en fases tempranas de vivero y de campo. La hipótesis de esta investigación fue que el roble, a pesar de ser una especie que crece naturalmente en suelos poco fértiles, tiene respuesta activa a la fertilización; en concordancia, la deficiencia en algún elemento nutricional genera patrones específicos de respuesta sobre sus características físicas. Este trabajo tuvo como objetivo identificar y describir los síntomas visuales de deficiencia de algunos nutrientes $(\mathrm{N}$, $\mathrm{P}, \mathrm{K}, \mathrm{Ca}, \mathrm{Mg}, \mathrm{S}$, y B) en plántulas de $Q$. humboldtii durante la etapa de vivero y establecer su relación con el contenido de nutrientes en los tejidos foliares.

\section{MATERIALES Y MÉTODOS}

El experimento se estableció en la Estación Forestal Experimental de Piedras Blancas (Universidad Nacional de Colombia, Sede Medellín), localizada en el corregimiento de Santa Elena, municipio de Medellín (6015'38" N y 75³0'23" W). El lugar está a una altitud de $2400 \mathrm{~m}$, con temperatura media anual de $15^{\circ} \mathrm{C}$, precipitación promedia anual de $1815 \mathrm{~mm}$ con régimen bimodal y humedad relativa media anual de $83 \%$ (León et ál. 2011). Las condiciones bioclimáticas del área corresponden a la zona de vida de bosque húmedo Montano Bajo (bh-MB) (Holdridge 1987).

El suelo utilizado como sustrato de crecimiento correspondió al horizonte superficial Bw $(30-50 \mathrm{~cm})$ de un Andisol de la Estación Forestal Experimental de Piedras Blancas el cual se encontraba bajo cobertura de pasto kikuyo sin fertilización (Cuadro 1). El suelo se clasificó 
Cuadro 1. Características químicas y físicas del sustrato de crecimiento de las plántulas de $Q$. humboldtii durante el ensayo antes y después de la fertilización aplicada en el tratamiento completo (Horizonte $B w$ de un Andisol bajo cobertura de pastos, Estación Forestal Experimental de Piedras Blancas).

\begin{tabular}{lcc}
\hline \multicolumn{1}{c}{ Parámetro } & Suelo testigo (T0) & Suelo con tratamiento completo (TC) \\
\hline Textura & Arena 92\%, limo 4\%, arcilla 4\% & - \\
$\mathrm{pH}$ & 5,0 & 5,4 \\
$\mathrm{MO}(\%)$ & 12,0 & 11,5 \\
$\mathrm{Al}\left(\mathrm{cmol}(+) \cdot \mathrm{kg}^{-1}\right)$ & 0,2 & 0,18 \\
$\mathrm{~N}\left(\mathrm{~g} \cdot \mathrm{kg}^{-1}\right)$ & 4,0 & 5,0 \\
$\mathrm{P}\left(\mathrm{mg} \cdot \mathrm{kg}^{-1}\right)$ & 1,0 & 6,0 \\
$\mathrm{Ca}\left(\mathrm{cmol}(+) \cdot \mathrm{kg}^{-1}\right)$ & 1,6 & 5,7 \\
$\mathrm{~K}\left(\mathrm{cmol}(+) \cdot \mathrm{kg}^{-1}\right)$ & 0,21 & 0,35 \\
$\mathrm{Mg}\left(\mathrm{cmol}(+) \cdot \mathrm{kg}^{-1}\right)$ & 0,34 & 0,8 \\
$\mathrm{~B}\left(\mathrm{mg} \cdot \mathrm{kg}^{-1}\right)$ & 0,6 & 0,8 \\
\hline
\end{tabular}

Métodos utilizados en los análisis: Textura: Bouyoucos. pH: Potenciométrico. Suelo: agua. 1:2. MO: Walkley-Black. Volumetría. Al: Cloruro de potasio 1M. Volumetría. N: Micro- Kjeldahl. P: Bray 2. Método azul de molibdato. Espectrofotómetro UV- VIS. K, Ca, Mg: Acetato de amonio 1M. Neutro. Absorción atómica. B: Agua caliente. Azometina - H. Espectrofotómetro UV- VIS.

como un Typic melanudand, el cual se caracteriza por formarse a partir de cenizas volcánicas y presentar alofana como material dominante en la fracción arcillosa, alto contenido de materia orgánica (>20\%), baja densidad aparente $\left(0,6 \mathrm{Mg} \mathrm{m}^{3}\right)$, muy alta capacidad de fijación fosfato aplicado (>99\%), extrema acidez (pH 4,7-4,9), baja concentración de calcio y magnesio intercambiables, media a alta disponibilidad de $\mathrm{K}$ intercambiable debido a que en el material parental hay alta cantidad de feldespato de potasio, baja disponibilidad de sulfato y boro y media a alta disponibilidad de hierro, manganeso, cobre y zinc (Osorio 2008). La muestra del suelo se secó al aire y se pasó a través de un tamiz con una malla de $4 \mathrm{~mm}$.

Las semillas de roble colectadas en los bosques naturales que rodean la Estación Forestal Experimental se desinfectaron mediante inmersión en hipoclorito de sodio al $2 \%$ durante 5 minutos y se lavaron con abundante agua destilada. Luego se sembraron en arena esterilizada en autoclave $\left(120^{\circ} \mathrm{C}, 20 \mathrm{~min}\right) \mathrm{y}$ se regaron diariamente. Dos meses después de la siembra, las plántulas recién germinadas se trasplantaron a tubetes (10 cm diámetro x $20 \mathrm{~cm}$ de profundidad) con un sustrato compuesto por turba y cascarilla de arroz (1:1), donde se dejaron durante 30 días más. Esto con el fin de promover una buena formación de raíces y permitir que con el tiempo, se agoten las reservas de nutrientes de las semillas. Finalmente, se seleccionaron plántulas con altura similar y con el mismo número de hojas y se trasplantaron a bolsas plásticas negras (15 cm de ancho x $30 \mathrm{~cm}$ de alto) que contenían 2,8 $\mathrm{kg}$ del sustrato utilizado para el ensayo. A cada bolsa con sustrato se le aplicaron $50 \mathrm{~g}$ de inóculo comercial de una mezcla de hongos formadores de micorrizas arbusculares (Glomus sp. Acaulospora sp., y Entrophospora sp.) y $50 \mathrm{~g}$ de inóculo natural tomado de robledales, el cual consistió en hojarasca tomada alrededor de árboles maduros de roble para aportar al sustrato de crecimiento los hongos formadores de ectomicorrizas (Amanita muscaria, Amanita sp. Suillus luteus) que 
requiere el roble. Después de la siembra de las plántulas el suelo se regó con agua destilada y a lo largo del ensayo se mantuvo entre 50 y $60 \%$ de su capacidad máxima de retención de agua.

Las fuentes de nutrientes se aplicaron al sustrato de cada bolsa con la siguiente secuencia: inicialmente se preparó una solución de ácido fosfórico que se adicionó con gotero al sustrato y se mezcló para garantizar una distribución uniforme. Por supuesto, esta aplicación inicial no se hizo en los tratamientos T0 y -P. Luego se llenó cada bolsa con el sustrato, se sembraron las plántulas y se aplicaron los demás nutrientes disueltos en $200 \mathrm{~cm}^{3}$ de agua destilada, según el tratamiento correspondiente (Cuadro 2). Finalmente, se adicionaron algunos microelementos (EDTA de $\mathrm{Fe}$, EDTA de Zn, EDTA de Cu) como fertilizante foliar, para evitar que se presentaran deficiencias por estos elementos no evaluados, lo cual podría interferir posteriormente con los resultados.

Cuadro 2. Fuentes de nutrientes y cantidades aplicadas al sustrato según el tratamiento de fertilización correspondiente. TC $=$ Completo, $-\mathrm{N}=\mathrm{TC}$ menos $\mathrm{N},-\mathrm{P}=\mathrm{TC}$ menos $\mathrm{P},-\mathrm{K}=\mathrm{TC}$ menos $\mathrm{K},-\mathrm{Ca}=\mathrm{TC}$ menos $\mathrm{Ca},-\mathrm{Mg}=\mathrm{TC}$ menos $\mathrm{Mg},-\mathrm{S}=$ TC menos $\mathrm{S},-\mathrm{B}=\mathrm{TC}$ menos $\mathrm{B}, \mathrm{T} 0=$ tratamiento testigo.

\begin{tabular}{|c|c|c|c|c|c|c|c|c|c|c|}
\hline \multicolumn{2}{|c|}{ Fuente } & \multicolumn{9}{|c|}{ Tratamiento } \\
\hline Urea & $\mathrm{CO}\left(\mathrm{NH}_{2}\right)_{2}(\mathrm{~g})$ & $\frac{\mathrm{TC}}{0,17}$ & $\frac{-\mathrm{N}}{-}$ & $\frac{-\mathrm{P}}{0,17}$ & $\frac{-\mathrm{K}}{0,17}$ & $\frac{-\mathrm{Ca}}{0,62}$ & $\frac{-\mathrm{Mg}}{-}$ & $\frac{-S}{0,17}$ & $\frac{-\mathrm{B}}{0,17}$ & $\begin{array}{r}\text { T0 } \\
-\end{array}$ \\
\hline Cloruro de potasio & $\mathrm{KCl}(\mathrm{g})$ & 0,42 & 0,42 & 0,42 & - & 0,42 & 0,42 & 0,42 & 0,42 & - \\
\hline Nitrato de calcio & $\mathrm{Ca}\left(\mathrm{NO}_{3}\right)_{2} 4 \mathrm{H}_{2} \mathrm{O}(\mathrm{g})$ & 1,75 & & 1,75 & 1,75 & - & 1,75 & 1,75 & 1,75 & - \\
\hline Sulfato de magnesio & $\mathrm{MgSO}_{4} 7 \mathrm{H}_{2} \mathrm{O}(\mathrm{g})$ & 0,70 & 0,70 & 0,70 & 0,70 & 0,70 & - & - & 0,70 & - \\
\hline Cloruro de magnesio & $\mathrm{MgCl}_{2} \cdot 6 \mathrm{H}_{2} \mathrm{O}(\mathrm{g})$ & - & - & - & - & - & - & 0,63 & - & - \\
\hline Cloruro de calcio & $\mathrm{CaCl}_{2} \cdot 2 \mathrm{H}_{2} \mathrm{O}(\mathrm{g})$ & - & 1,09 & - & - & - & - & - & - & - \\
\hline Sulfato de amonio & $\left(\mathrm{NH}_{4}\right)_{2} \cdot \mathrm{SO}_{4}(\mathrm{~g})$ & - & - & - & - & - & 0,38 & - & - & - \\
\hline Solubor & $\mathrm{Na}_{2} \mathrm{~B}_{8} \mathrm{O}_{13} \cdot 4 \mathrm{H}_{2} \mathrm{O}(\mathrm{g})$ & 0,01 & 0,01 & 0,01 & 0,01 & 0,01 & 0,01 & 0,01 & - & - \\
\hline Acido fosfórico & $\mathrm{H}_{3} \mathrm{PO}_{4}(85 \%)\left(\mathrm{cm}^{3}\right)$ & 2,40 & 2,40 & - & 2,40 & 2,40 & 2,40 & 2,40 & 2,40 & - \\
\hline
\end{tabular}

Se registraron las características de los síntomas de deficiencia en las plantas. Luego, se escogieron hojas representativas de cada tratamiento de elemento faltante en las que se expresaron los síntomas de deficiencias correspondientes, y se compararon con hojas de las plántulas con fertilización completa. Se determinó el color de las hojas (tabla Munsell para tejidos vegetales), la forma, textura, presencia de manchas, clorosis y necrosis, entre otras.
Para la realización de los análisis químicos foliares se colectaron 3 muestras combinadas de hojas nuevas pero completamente desarrolladas por tratamiento, cada una de aproximadamente $20 \mathrm{~g}$ (base húmeda); las hojas correspondieron al segundo verticilo contadas a partir del ápice del eje principal. Las muestras se secaron en un horno de circulación forzada $\left(65^{\circ} \mathrm{C}, 72 \mathrm{~h}\right)$ y se molieron (molino marca Willy). Luego se tomaron $3 \mathrm{~g}$ de tejido foliar seco y se aplicaron las siguientes técnicas para determinar 
el contenido de elementos: N: Micro-Kjeldahl (NTC668); P: Acido L-ascórbico, Colorimétrica (NTC5350); Ca, Mg, K: Absorción atómica y digestión vía seca (NTC-5151); B: Azometina-H y colorimétrico.

Para evaluar el efecto de los diferentes tratamientos de fertilización sobre los contenidos foliares de nutrientes, se practicaron análisis de varianza con prueba $\mathrm{F}(\mathrm{p}=0,05)$. Cuando la $\mathrm{F}$ fue significativa, se aplicó la prueba de Tukey $(\mathrm{p}=0,05)$ para determinar las diferencias entre tratamientos. Se utilizó el programa estadístico Statgraphics Centurion XVI.

\section{RESULTADOS Y DISCUSIÓN}

\section{Síntomas visuales de deficiencias nutricionales}

Los tratamientos de fertilización aplicados a las plántulas, determinaron la aparición de síntomas visuales evidentes de deficiencia nutricional. Estos síntomas comenzaron a expresarse a partir del segundo mes de establecido el ensayo, pero se hicieron completamente notorios al cuarto mes. El efecto de los tratamientos se empezó a hacer evidente sobre la altura de las plántulas, y después aparecieron diferencias en la forma de las plántulas, y en algunas características de las hojas como forma, tamaño, textura y color. Además, se presentaron otros síntomas específicos, relacionados con la forma de las quemazones y los tipos de clorosis, entre otros (Figuras 1 y 2 ). La mayoría de estos síntomas visuales de deficiencias nutricionales se han reportado para otras especies forestales; no obstante, cada especie puede mostrar tales deficiencias de forma particular y con diferente intensidad (Sarcinelli et ál. 2004, Wallau et ál. 2008).

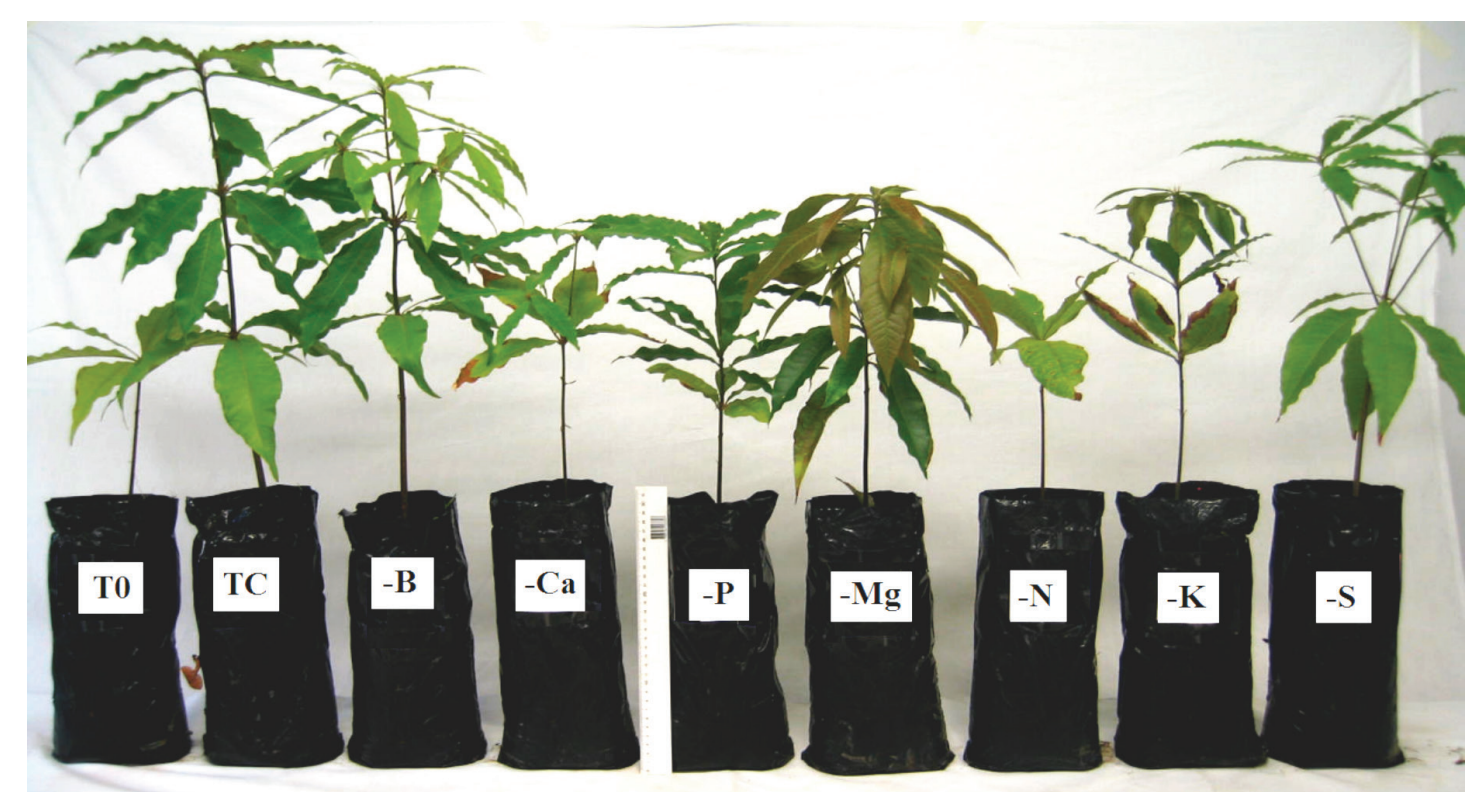

Fig. 1. Aspecto de las plántulas de $Q$. humboldtii después de 4 meses bajo tratamientos de fertilización en vivero: T0= tratamiento testigo (sin fertilización), TC= completo (con B, Ca, P, Mg, N, K y S), -B=TC menos B, - Ca=TC menos $\mathrm{Ca},-\mathrm{P}=\mathrm{TC}$ menos $\mathrm{P},-\mathrm{Mg}=\mathrm{TC}$ menos $\mathrm{Mg},-\mathrm{N}=\mathrm{TC}$ menos $\mathrm{N},-\mathrm{K}=\mathrm{TC}$ menos $\mathrm{K},-\mathrm{S}=\mathrm{TC}$ menos $\mathrm{S}$. 
A

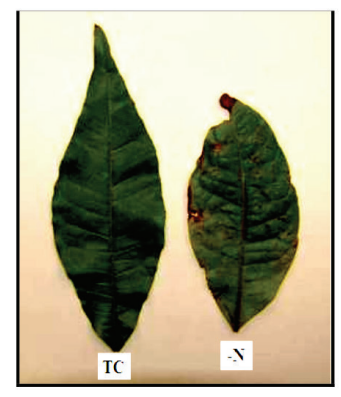

E

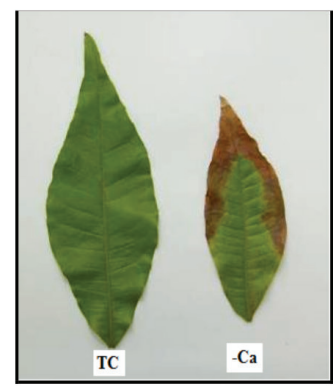

B

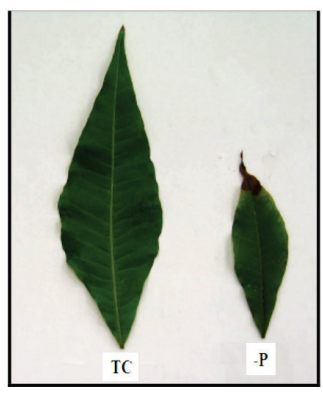

$\mathrm{F}$

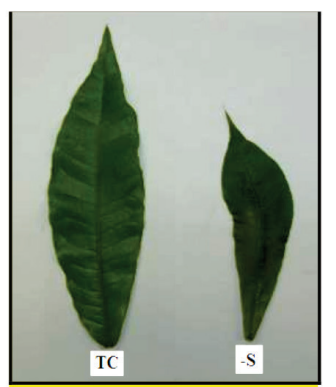

C

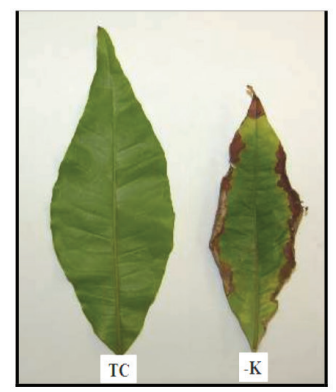

G

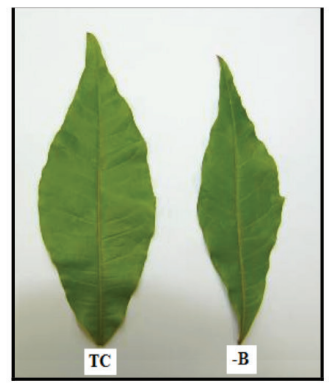

D

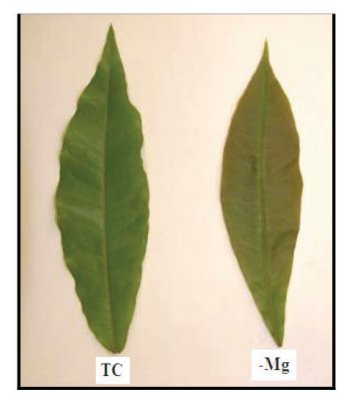

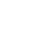
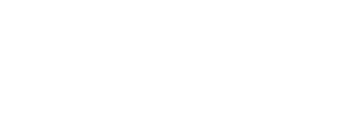

\section{.} Fig. 2. Comparación entre hojas de plántulas de $Q$. humboldtii con tratamiento completo y hojas provenientes de A: $-\mathrm{N}=\mathrm{TC}$
menos nitrógeno, $\mathrm{B}:-\mathrm{P}=\mathrm{TC}$ menos fósforo, $\mathrm{C}:-\mathrm{K}=\mathrm{TC}$ menos potasio, $\mathrm{D}:-\mathrm{Mg}=\mathrm{TC}$ menos $\mathrm{magnesio}, \mathrm{E}:-\mathrm{Ca}=\mathrm{TC}$ menos calcio, $\mathrm{F}$ : $-\mathrm{S}=\mathrm{TC}$ menos azufre, $\mathrm{G}$ : $-\mathrm{B}=\mathrm{TC}$ menos boro.

Síntomas de deficiencia de N. Las plántulas de $Q$. humboldtii deficientes en $\mathrm{N}$ tuvieron un fuerte retraso en su crecimiento, pues solamente desarrollaron un verticilo, al igual que las del tratamiento T0 (Figura 1). En comparación con hojas de plántulas con tratamiento completo, éstas fueron más pequeñas y de color verde más claro $(2,5 \mathrm{GY} 6 / 8$, tabla Munsell de tejidos vegetales), con moteados y nervaduras amarillentas, puntas quemadas, parches de quemazón y textura más coriácea (Figura 2A). Resultados similares en desarrollo de plántulas y hojas deficientes en $\mathrm{N}$ se presentaron en Tectona grandis (Barroso et ál. 2005) y Eucalyptus citriodora (Maffeis et ál. 2000).

La reducción en el crecimiento por la deficiencia de $\mathrm{N}$ se debió probablemente a que este elemento es necesario para la síntesis de proteínas
(Raij 1991) y la inhibición de la síntesis reduce el proceso de división celular, que afecta el crecimiento de la planta (Malavolta 2006). Por esto, las plántulas no generaron nuevas hojas, y las que ya se habían formado antes de la aplicación del tratamiento, mostraron síntomas de deficiencia puesto que la cantidad de $\mathrm{N}$ no fue suficiente para un total desarrollo. Esta inhibición del crecimiento se ha observado cuando la carencia de $\mathrm{N}$ es muy extrema (Marques et ál. 2004). Así mismo, la coloración verde claro y amarillenta se podría explicar por la reducción de la síntesis de clorofila (Fabasi y Viégas 1997).

Síntomas de deficiencia de P. Las plántulas deficientes en $\mathrm{P}$ presentaron un retraso leve de crecimiento, puesto que produjeron 3 verticilos pero con entrenudos más cortos que los del 
tratamiento TC (Figura 1). Las hojas fueron de menor tamaño, pero no se observaron diferencias importantes en color y forma comparadas con las hojas de plántulas no deficientes (5GY4/8, tabla Munsell de tejidos vegetales), aunque se presentaron algunas marcas de quemazón hacia los bordes (Figura 2B). La deficiencia de $\mathrm{P}$ no presentó síntomas tan marcados y generalizados como la de N. Resultados similares en la apariencia de las plántulas y las hojas deficientes en P se obtuvieron en Schizolobium amazonicum y Hevea brasiliensis (Marques et ál. 2004), mientras que en otras especies forestales se han registrado síntomas más fuertes de deficiencia en $\mathrm{P}$, como poca rigidez de la planta, color más oscuro, enrojecimiento del tejido y quemazones severas de la lámina foliar, entre otros (Camargos et ál. 2002, Coelho et ál. 2002, Sarcinelli et ál. 2004).

Las especies arbóreas generalmente se caracterizan por una alta eficiencia en la absorción de $\mathrm{P}$ en su fase inicial de crecimiento (Sarcinelli et ál. 2004), particularmente cuando el sustrato se inocula con hongos formadores de micorrizas, lo cual podría explicar que el efecto de su deficiencia no fuera tan marcado. El P en las plantas tiene un papel importante en la producción de energía, por lo cual es probable que su ausencia se refleje en un menor crecimiento (Taiz y Zaiger 1998), como efectivamente sucedió en las plántulas de roble.

Síntomas de deficiencia de K. Las plántulas de roble deficientes en $\mathrm{K}$ fueron de menor tamaño y biomasa que las del TC. Al igual que el tratamiento -P, las plántulas formaron 3 verticilos pero con entrenudos cortos (Figura 1) y las hojas en general fueron más pequeñas. Las hojas más viejas fueron las que más rápidamente mostraron síntomas de deficiencia como lo reportado por Malavolta 1980, que comprendieron hojas de colores más claros $(5 \mathrm{GY} 4 / 6)$ con puntos cloróticos que se extendieron desde los bordes hacia el centro por entre las nervaduras, y luego se desarrolló una necrosis en el borde con enrollamiento de la parte afectada (Figura 2C). Esto coincide con los síntomas reportados en Theobroma grandiflorum (Salvador et ál. 1994) y Swietenia macrophylla (Wallau et ál. 2008).

Se han reportado otros síntomas de deficiencia de $\mathrm{K}$ un poco diferentes a los aquí encontrados, tales como clorosis intervenal generalizada y presencia de puntos necróticos en las hojas viejas (Barroso et ál. 2005 y Días et ál. 1991). El primer síntoma de deficiencia de K en plántulas de $Q$. humboldtii fue la reducción de la tasa de crecimiento y luego la expresión de los síntomas en las hojas, lo cual coincide con lo reportado por Mengel y Kirkby (1987) y Marschner (1995).

Síntomas de deficiencia de Mg. Las plántulas deficientes en Mg fueron de menor tamaño comparadas con las del TC, y al igual que para los tratamientos -P y -K, formaron 3 verticilos pero con entrenudos más cortos (Figura 1). Las hojas viejas se tornaron de un color verde más claro y rojizo (5GY4/6), con algunos síntomas de clorosis intervenal y en las puntas de la lámina foliar; también se presentó necrosis hacia el ápice y se encontraron bordes retorcidos (Figuras 2D). Estos mismos resultados se reportaron para Acacia holosericea (Sarcinelli et ál. 2004), Bertholletia excelsa (Camargos et ál. 2002) y Tectona grandis (Barroso et ál. 2005).

Malavolta (1980) propuso que esta clorosis se debe a la reducción en la cantidad de clorofila ya que el $\mathrm{Mg}$ es un componente de esta molécula (Mengel y Kirkby 1987), y su deficiencia reduce la fotosíntesis, provoca clorosis y afecta el crecimiento de las plantas. El Mg se traslada fácilmente a las regiones de crecimiento activo $\mathrm{y}$, como resultado, los síntomas de deficiencia aparecen primero en las hojas maduras, que avanzaron hacia las hojas nuevas (Bould et ál. 1983), tal como ocurrió en $Q$. humboldtii.

Síntomas de deficiencia de Ca. Las plántulas deficientes en $\mathrm{Ca}$ presentaron reducción en el crecimiento en altura comparadas con las del TC, y formaron solamente 2 verticilos (Figura 1), lo cual se ajusta a los síntomas reportados por Malavolta (2006). Tanto las hojas nuevas como 
las viejas fueron más pequeñas, exhibieron un color verde amarillento (2,5GY6/8) principalmente hacia el margen, y una quemazón muy típica desde el ápice, que se extiende por el margen (Figura 2E). Estos síntomas también fueron reportados en Aspidosperma polyneuron (Muniz y Silva 1995), Theobroma grandiflorum (Salvador et ál. 1994), Swietenia macrophylla (Wallau et ál. 2008) y Bertholletia excelsa (Camargos et ál. 2002). Algunos autores como Sarcinelli et ál. (2004), Santi et ál. (2006) y Marques (2004) encontraron síntomas adicionales como deformaciones muy notorias en las hojas nuevas de árboles con deficiencia en $\mathrm{Ca}$. Estos cambios en la estructura se presentan debido a que este elemento es esencial para la constitución de la pared celular, la cual define parte del transporte en la membrana.

Síntomas de deficiencia de S. Las plantas deficientes en $\mathrm{S}$ tuvieron una leve disminución en altura, al igual que el tratamiento $-\mathrm{Ca}$, y formaron solamente 2 verticilos (Figura 1). Las hojas presentaron una forma más lanceolada y a veces un tamaño mayor que las hojas de plantas sin deficiencias, así como un color verde más oscuro (5GY4/8). Las hojas nuevas fueron más delgadas y presentaron una textura papelosa, por lo cual caían péndulas en la plántula. Algunas hojas presentaron asimetría de la lámina con respecto a la nervadura central (Figura 2F). En Tectona grandis también se observó la reducción en el crecimiento por la carencia de este nutriente
(Barroso et ál. 2005). Otros síntomas más típicos de la deficiencia de $\mathrm{S}$ son clorosis marcada y generalizada en las hojas nuevas (Wallau et ál. 2008, Sarcinelli et ál. 2004, Malavolta 2006). El $\mathrm{S}$ es un elemento que se encuentra en las plantas que forma principalmente proteínas con una baja redistribución interna, por lo cual los síntomas de deficiencia aparecen primero en las hojas nuevas, como se observó en $Q$. humboldtii.

Síntomas de deficiencia de B. En general, las plántulas a las que no se les aplicó B presentaron un tamaño similar al de las plántulas de tratamiento TC (Figura 1). Las hojas no mostraron diferencias marcadas en cuanto a color (5GY5/8) y tamaño. La principal diferencia se encontró en la forma de la lámina, ya que las hojas deficientes presentaron formas más alargadas y estrechas (Figuras 2G).

\section{Contenido foliar de nutrientes}

Los resultados del análisis de varianza para el contenido de nutrientes foliares indicaron que hubo un efecto altamente significativo $(\mathrm{p}<0,001)$ de los elementos N, P, K, Mg y B y un efecto significativo $(\mathrm{p} \leq 0,05)$ para el contenido foliar de $\mathrm{Ca}$ (Cuadro 3). La prueba de comparación de medias de Tukey (95\%) estableció que cada deficiencia inducida produjo una disminución significativa de la concentración del respectivo elemento con respecto al tratamiento completo (Cuadro 4).

Cuadro 3. Resultados del ANOVA para el contenido foliar de nutrientes en plántulas de Q. humboldii. Se presentan los valores $F$ y p. Los asteriscos indican el nivel de significancia de las diferencias entre las medias de los tratamientos $(* * *)=\mathrm{p} \leq 0,001,(* *)=\mathrm{p} \leq 0,01(*)=\mathrm{p} \leq 0,05$.

\begin{tabular}{cc}
\hline Contenido de nutrientes foliares $(\%)$ & Fertilización \\
\hline $\mathrm{N}$ & $F_{(8,18)}=8,19 ; \mathrm{p}=0,000^{* * *}$ \\
$\mathrm{P}$ & $F_{(8,18)}=14,43 ; \mathrm{p}=0,000 * * *$ \\
$\mathrm{~K}$ & $F_{(8,18)}=25,54 ; \mathrm{p}=0,000^{* * *}$ \\
$\mathrm{Mg}$ & $F_{(8,18)}=11,26 ; \mathrm{p}=0,000^{* * *}$ \\
$\mathrm{Ca}$ & $F_{(8,18)}=2,67 ; \mathrm{p}=0,0398^{*}$ \\
$\mathrm{~B}$ & $F_{(8,18)}=55,63 ; \mathrm{p}=0,000^{* * *}$ \\
\hline
\end{tabular}


SEPÚLVEDA et ál.: Deficiencias nutricionales de plántulas del roble andino

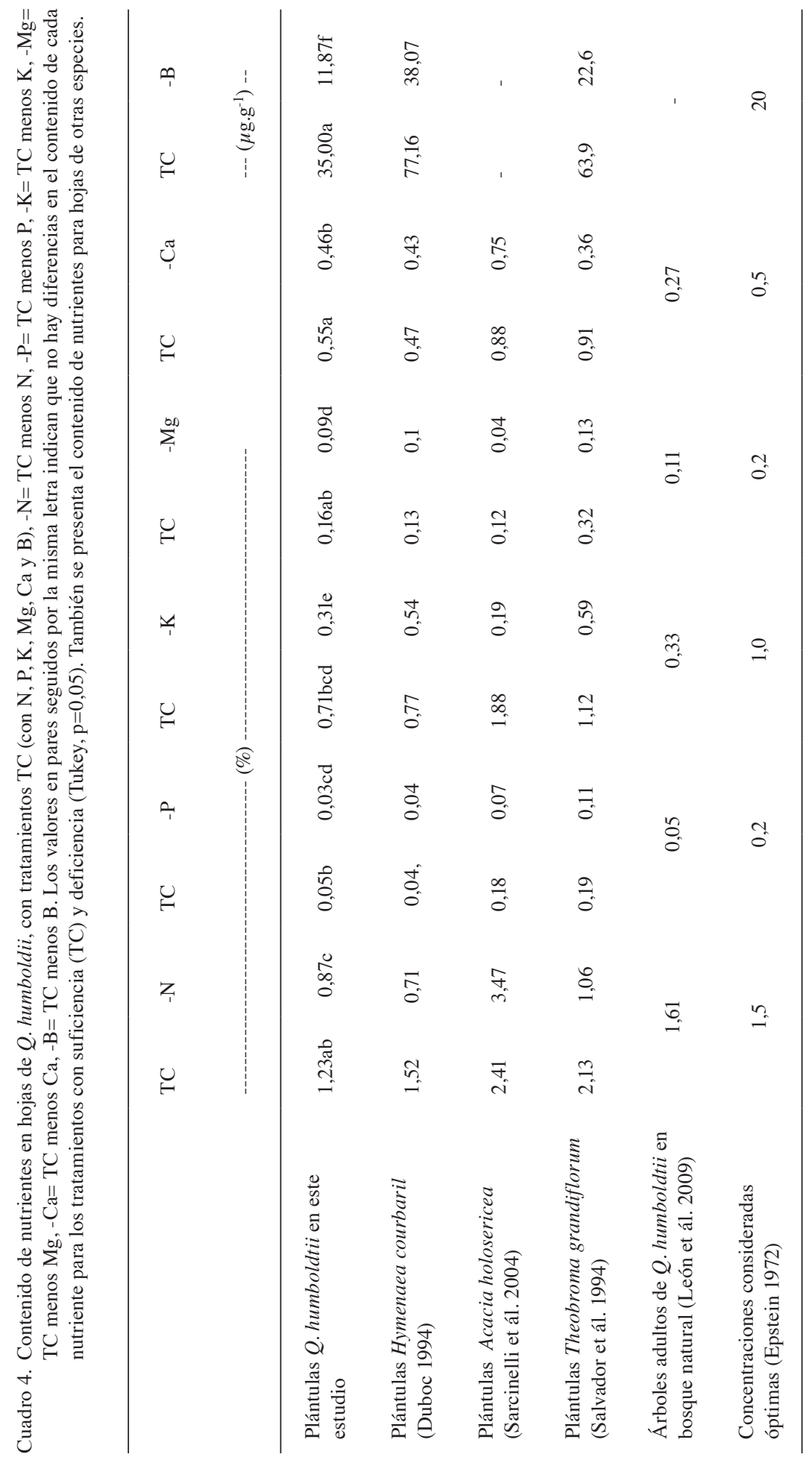

Agronomía Costarricense 38(1): 161-173. ISSN:0377-9424 / 2014 
Contenido foliar de N. La concentración foliar de $\mathrm{N}$ en plántulas que recibieron la fertilización completa fue 1,5 veces mayor que la de plántulas a las que no se sumnistró este elemento (-N) (Cuadro 4). Las concentraciones foliares de $\mathrm{N}$ en las plántulas de $Q$. humboldtii deficientes en este elemento, fueron similares a los obtenidos en Himenaea courbaril (Duboc 1994) y Theobroma grandiflorum (Salvador et ál. 1994). La concentración de $\mathrm{N}$ en plántulas de roble con tratamiento TC fue ligeramente inferior a la de árboles adultos en bosques naturales de Antioquia (León et ál. 2009) (Cuadro 4).

Contenido foliar de P. El tratamiento -P presentó valores de concentración foliar de $\mathrm{P}$ estadísticamente inferiores a los del tratamiento TC (Cuadro 4), resultado contrastante al obtenido con plántulas de Theobroma grandiflorum (Salvador et ál. 1994), sometidas a los tratamientos TC y -P, que presentaron mayores concentraciones de $\mathrm{P}$ (más del doble). Sin embargo, mantuvieron una proporción similar de $\mathrm{P}$ a la aquí encontrada en $Q$. humboldtii. El contenido foliar de $\mathrm{P}$ en plántulas fertilizadas completamente (TC) fue casi el doble al de las plántulas sin suministro de este nutriente. Concentraciones foliares similares de $\mathrm{P}$ fueron reportadas por Duboc (1994) para plántulas de Hymenaea courbaril y por León et ál. (2009) para árboles adultos de $Q$. humboldtii.

Contenido foliar de K. La concentración foliar de $\mathrm{K}$ en plántulas con fertilización completa fue más del doble que la de plántulas no fertilizadas (-K), y presentan diferencias estadísticamnete significativas (Cuadro 4). Concentraciones foliares similares a las aquí obtenidas al evaluar la deficiencia de $\mathrm{K}$, fueron reportadas por Duboc (1994) y Santi et ál. (2006) para Hymenaea courbaril. Para ésta y otras especies se encontró que la concentración de $\mathrm{K}$ en tratamientos con suficiencia (TC) fue superior a la encontrada en $Q$. humboldtii (Duboc 1994, Santi et ál. 2006, Salvador et ál. 1994 y Sarcinelli et ál. 2004). La concentración de $\mathrm{K}$ en hojas de árboles adultos de $Q$. humboldtii encontrada por León et ál. (2009) fue similar a la reportada en este trabajo para plántulas a las que no se suministró el elemento (Cuadro 4).

Contenido foliar de Mg. El contenido foliar de $\mathrm{Mg}$ en plántulas fertilizadas completamente fue casi del doble en comparación con plántulas no fertilizadas (Cuadro 4). Los valores de concentración de $\mathrm{Mg}$ en el tratamiento deficiente fueron similares a los encontrados por Duboc (1994) en Hymenaea courbaril y por Salvador et ál. (1994) en Theobroma grandiflorum (Cuadro 4). Así mismo, la concentración de $\mathrm{Mg}$ en las hojas de roble con tratamiento completo fue similar a la encontrada por Duboc (1994) en Hymenaea courbaril y por Sarcinelli et ál. (2004) en Acacia holosericea, pero otros autores encontraron concentraciones superiores de $\mathrm{K}$ en tejidos foliares: Salvador et ál. (1994) en Theobroma grandiflorum y León et ál. (2009) en árboles adultos de $Q$. humboldtii (Cuadro 4).

Contenido foliar de Ca. El contenido foliar de $\mathrm{Ca}$ en las plántulas con fertilización completa (TC) fue levemente superior al de las plántulas no fertilizadas (-Ca) (Cuadro 4). Las concentraciones foliares correspondientes a este útimo tratamiento fueron intermedias con respecto a otros reportes. Así, fueron similares a los obtenidos para especies como Hymenaea courbaril y Theobroma grandiflorum (Duboc 1994, Salvador et ál. 1994), muy superiores a los hallados por Sarcinelli et ál. (2004) para Acacia holosericea. La concentración foliar de $\mathrm{Ca}$ en plántulas de roble bajo tratamiento TC fue similar a la encontrada por Duboc (1994) en Hymenaea courbaril, e inferior a las encontradas para las especies Sorghum bicolor (Santi et ál. 2006), Theobroma grandiflorum (Salvador et ál. 1994) y Acacia holosericea (Sarcinelli et ál. 2004). Por otra parte, las concentraciones foliares de Ca correspondientes al tratamiento $-\mathrm{Ca}$, fueron superiores a las reportadas para árboles adultos de $Q$. humboldtii en bosques 
altoandinos (León et ál. 2009), como resultado seguramente de la alta acidez de los suelos presentes en estos últimos.

Contenido foliar de B. El contenido foliar de B en plántulas fertilizadas completamente fue casi 3 veces al obtenido en plántulas no fertilizadas (-B). El B no se presentó como un elemento limitante para el desarrollo de las plántulas de roble. El análisis foliar determinó que aunque las plántulas deficientes en B presentaron la menor concentración de este elemento en las hojas, la concentración de B en las hojas del tratamiento TC también fue baja en comparación con los otros tratamientos. El B se considera un elemento traza, así que se requiere muy poco para satisfacer su demanda. Al parecer la semilla de roble o el suelo contaban con la concentración necesaria para suplir esta demanda en la fase de vivero y en consecuencia, las plántulas no mostraron deficiencias (Cuadro 4).

Las concentraciones de $\mathrm{K}, \mathrm{Mg}$ y $\mathrm{Ca}$ en las hojas de $Q$. humboldtii provenientes del tratamiento con fertilización completa (TC) fueron cercanas a las que se han considerado como adecuadas para las plantas según Epstein (1972) (Cuadro 4). De manera similar, estas coinciden con el rango adecuado (o al menos suficiente) reportado para especies de interés agronómico, tal como lo reportan Guerrero (1988) e ICA (1992). En consecuencia, se podría considerar que las concentraciones de estos elementos en el suelo fueron adecuadas.

El caso especial del $\mathrm{P}$, que presentó concentraciones foliares muy bajas en las plántulas de roble, incluso en aquellas provenientes del tratamiento TC $(<0,15 \%)$, se puede asociar con la baja concentración de este elemento aún en el suelo fertilizado $\left(6 \mathrm{mg} \cdot \mathrm{kg}^{-1}\right)$. Esto indica que la cantidad de $\mathrm{P}$ aplicada al suelo fue inferior a la requerida para un óptimo desarrollo de las plántulas que para muchas especies de interés agronómico está entre $15-30$ mg. $\mathrm{kg}^{-1}$ (P extraído por el método de Bray-II). La alta fijación del $\mathrm{P}$ en suelos derivados de ceniza volcánica (León et ál. 2011), como el usado en este ensayo, puede ser la razón de la baja disponibilidad de este elemento para las plántulas. En estos suelos el fosfato es adsorbido por la partículas de la fracción arcillosa (alofana, goetita) tan fuertemente que no pasa a la solución del suelo (Bohn et ál. 1985, Bolan 1991) que restringe así, la toma de $\mathrm{P}$ por las plantas y por los mismos hongos micorrizales (Osorio 2012). Al parecer la asociación micorrizal no fue suficientemente efectiva para aumentar la absorción de este elemento hasta un nivel óptimo debido a la baja disponibilidad del elemento (Habte y Manjunath 2001). La baja concentración foliar de $\mathrm{P}$ en árboles adultos de $Q$ humboldtii que crecen en Andisoles en un área cercana a la zona de estudio reportada por León et ál. (2009) confirman la baja disponibilidad del $\mathrm{P}$ en los suelos de esta zona (Cuadro 4).

En las hojas de plántulas de Q. humboldtii se presentó una relación $\mathrm{P}: \mathrm{N}=0,038$, que aunque fue mayor que a la encontrada por León et ál. (2009), indica una baja disponibilidad de $\mathrm{P}$ en el suelo (Medina et ál. 1990). Inversamente, la relación N:P en este estudio fue de 26,7 , muy superior al valor 16 considerado como adecuado por este autor, lo que sugiere que hay una deficiencia crítica de $\mathrm{P}$ con respecto a $\mathrm{N}$. La relación $\mathrm{N}: \mathrm{Mg}=7,69$ fue muy inferior a 17,5 (Zas y Serrada 2003), considerado como un valor que garantiza un suministro adecuado de $\mathrm{Mg}$, sugiere que el sustrato fue adecuado al no detectarse deficiencias críticas de $\mathrm{Mg}$ con respecto a $\mathrm{N}$ a nivel foliar. El valor de la relación $\mathrm{K}: \mathrm{Ca}=1,29$ también fue superior al umbral de 0,5 sugerido por Ballard y Carter (1986) como indicativo de suficiencia en la nutrición de K.

Con los datos anteriores se proponen, de manera tentativa, rangos aproximados de concentración foliar de nutrientes que pueden definir condiciones de suficiencia y deficiencia en plántulas de $Q$. humboldtii en la fase de vivero (Cuadro 5); no se tuvo en cuenta el P, ya que sus valores al parecer fueron bajos debido a la poca disponibilidad de este elemento en el suelo usado para el ensayo. 
Cuadro 5. Rangos de concentración foliar deficiente y suficiente de elementos en plántulas de $Q$. humboldtii en vivero.

\begin{tabular}{lccccc}
\hline $\begin{array}{l}\text { Concentraciones de elementos } \\
\text { en hojas }\end{array}$ & $\mathrm{N}$ & $\mathrm{K}$ & $\mathrm{Mg}$ & $\mathrm{Ca}$ & $\mathrm{B}$ \\
& & & & $\left(\mu \mathrm{g} . \mathrm{g}^{-1}\right)$ \\
\hline $\begin{array}{l}\text { Deficiente } \\
\text { Suficiente }\end{array}$ & $<1,1$ & $<0,4$ & $<0,09$ & $<0,35$ & $<12$ \\
\hline
\end{tabular}

\section{CONCLUSIONES}

La descripción de los síntomas visuales de deficiencia nutricional en especies como $Q$. humboldtii representa una herramienta valiosa para la determinación de problemas nutricionales asociados a concentraciones deficientes de nutrientes, tanto en tejidos como en el sustrato de crecimiento. La identificación de tales síntomas asociados a la deficiencia de cada nutriente, sirve para enfocar los programas de fertilización a los nutrientes que verdaderamente causan limitaciones para el desarrollo de las plántulas. No obstante, la intensidad de la fertilización solo se puede determinar con análisis químicos del contenido de nutrientes en tejidos y suelos.

Quercus humboldtii, al igual que otras especies forestales, presentó los síntomas visuales de deficiencia típicos para cada uno de los macroelementos evaluados; estos síntomas siempre estuvieron asociados con los menores valores de concentración foliar de cada nutriente, registrados en los tratamientos con el elemento faltante. Aunque la ausencia de Boro no generó síntomas visuales de deficiencia, sí determinó menores contenidos de este elemento en los tejidos foliares. La asociación de la concentración de nutrientes en los tejidos con su disponibilidad en el suelo y con el desarrollo de las plántulas, permite concluir que para todos los casos, excepto para el $\mathrm{P}$, la fertilización inicial fue suficiente para obtener un buen desarrollo vegetal. La disponibilidad tan baja de $\mathrm{P}$ en este suelo derivado de ceniza volcánica, probablemente determinó concentraciones muy bajas de este nutriente en las hojas.

\section{AGRADECIMIENTOS}

Este trabajo recibió apoyo financiero de la Universidad Nacional de Colombia y de CORANTIOQUIA a través del proyecto "Ecología y Silvicultura del roble de tierra fría (Quercus humboldtii Bonpl.). Contrato $\mathrm{N}^{\circ} .7203$ de 2006, así como de COLCIENCIAS a través del proyecto "Fertilización exponencial en especies forestales de los bosques andinos de Colombia" (Contrato 655 de 2008). Agradecemos al Posgrado en Bosques y Conservación Ambiental, al Laboratorio de Ecología César Pérez Figueroa y a la Estación Forestal Experimental de Piedras Blancas por su apoyo en aspectos logísticos del desarrollo de este trabajo.

\section{LITERATURA CITADA}

BALLARD R., CARTER R. 1986. Evaluating forest stand nutrient status. Land Management Report $\mathrm{N}^{\circ} 20$, Ministry of Forest, Victoria, BC. 32 p.

BARROSO D., FIGUEIREDO F., PEREIRA R., MENDONÇA A., SILVA L. 2005. Diagnóstico de deficiências de macronutrientes em mudas de Teca. Revista Árvore 29(5):671-679.

BOHN H., McNEAL B.L., O'CONNOR G. 1985. Soil Chemistry. John Wiley and Sons, New York, 341 p.

BOLAN N.S. 1991. A critical review on the role of mycorrhizal fungi in the uptake of phosphorus by plants. Plant Soil 134:189-207.

BOULD C., HEWITT E., NEEDHAM P. 1983. Diagnosis of mineral disorders in plants: principles. London: HMSO, V1, 174 p.

CAMARGOS S., MURAOKA T., FERNANDES S., SALVADOR J. 2002. Diagnose nutricional em mudas de castanheirado-Brasil. Revista Agricultura Tropical 6(1):81-96.

CARPANEZZI A., BRITO J., FERNANDES P., JARK FILHO W. 1999. Teor de macro e micronutrientes 
em folhas de diferentes idades de algumas essências florestais nativas. Anais da E.S.A. "Luiz de Queiroz" 23:225-232.

COELHO A., WAQUIL J., KARAN D., CASELA C., RIBAS P. 2002. Seja o doutor do seu sorgo. Piracicaba: POTAFOS Arquivo do agrônomo V.14, 24 p.

DÍAS L., ALVAREZ V., BRIENZA J. 1991. Formação de mudas de Acacia mangium II. Resposta à nitrogênio e potássio. Rev. Árvore 16(2):135-143.

DUBOC E. 1994. Nutrição do Jatobá (Hymenaea courbaril L., var. Stilbocarpa (Hayne) Lee et Lang.). Mestrado em Engenharia Florestal, Universidad Federal de Lavras, 90 p.

EPSTEIN E. 1972. Mineral nutrition of plants: principles and perspectives. John Wiley, New York, $412 \mathrm{p}$.

FABASI J, VIÉGAS I.J. 1997. Concentração de N, P, K, $\mathrm{Ca}, \mathrm{Mg}$ e $\mathrm{S}$ em plantas de malva (Urena lobata L.), cultivar BR-01, cultivadas em solução nutritiva com omissão de macro e micronutrientes. Boletim da Fundação de Ciências Agrárias do Pará (27):31- 42.

GUERRERO R. 1988. El diagnóstico químico de la fertilidad del suelo, pp. 151-210. In: F. Silva. (ed.) Fertilidad de Suelos, diagnóstico y control. Sociedad Colombiana de Ciencia del Suelo. Bogotá.

HABTE M., MANJUNATH A. 1991. Categories of vesiculararbuscular mycorrhizal dependency of host species. Mycorrhiza 1:3-12.

HOLDRIDGE L.R. 1987. Ecología Basada en Zonas de Vida. Instituto Interamericano de Cooperación para la Agricultura, San José, Costa Rica, 216 p.

INSTITUTO COLOMBIANO AGROPECUARIO -ICA. 1992. Fertilización en diversos cultivos, $5^{\text {a }}$ Aproximación. Manual de asistencia técnica $\mathrm{N}^{\circ} .25$. ICA, Bogotá, 64 p.

JARAMILLO D. 1995. Andisoles del oriente antioqueño. Caracterización química y fertilidad. Universidad Nacional de Colombia, Medellín, 35 p.

LEÓN J., GONZÁleZ M., GALLARDO J. 2009. Retranslocación y eficiencia en el uso de nutrientes en bosques del centro de Antioquia. Revista Colombia Forestal 12:119-140.

LEÓN J., GONZÁLEZ M., GALLARDO J. 2011. Ciclos biogeoquímicos en bosques naturales y plantaciones de coníferas en ecosistemas de alta montaña de Colombia. Revista Biología Tropical 59 (4):18831894.

MAFFEIS A., SILVEIRA R., BRITO J. 2000. Reflexos das deficiências de macronutrientes e boro no crescimento de plantas, produção e qualidade de óleo essencial em Eucalyptus citriodora. Scientia Forestalis 57:87-98.

MALAVOLTA E. 1980. Elementos de nutrição mineral de plantas. Agronômica Ceres, São Paulo, 251 p.
MALAVOLTA E. 2006. Manual de nutrição mineral de plantas. Livroceres, São Paulo, 638 p.

MARQUES T., GUEDES J., PINTO M., FERREIRA P. 2004. Crescimento inicial do paricá (Schizolobium amazonicum) sob omissão de nutrientes e de sódio em solução nutritiva. Cerne 10(2):184-195.

MARSCHNER H. 1995. Mineral nutrition of higher plants. Academic Press, London. 889 p.

MEDINA E., GARCÍA V., CUEVAS E. 1990. Sclerophylly and oligotrophy environments: relationships between leaf structure, mineral nutrient content, and drought resistance in tropical rain forest of upper Rio Negro region. Biotropica (22):51-64.

MENGEL K. KIRKBY E. 1987. Principles of plant nutrition. International Potash Institute, Bern, $687 \mathrm{p}$.

MUNIZ A.S., SILVA M. 1995. Exigências nutricionais de mudas de Peroba Rosa (Aspidosperma polyneurom) em solução nutritiva. Revista Árvore 19:263-271.

OSORIO N.W. 2008. Effectiveness of microbial solubilization of phosphate in enhancing plant phosphate uptake in tropical soils and assessment of the mechanisms of solubilization. Ph.D. dissertation, University of Hawaii, Honolulu, 358 p.

OSORIO N.W. 2012. Manejo de nutrientes en suelos del trópico. Universidad Nacional de Colombia, Medellín, $345 \mathrm{p}$

RAIJ B. 1991. Fertilidade do solo e adubação. Agronômica Ceres/Potafos, Piracicaba, 343 p.

SALVADOR J., MURAOKA T., ROSSETO R., RIBEIRO G.A. 1994. Sintomas de deficiências nutricionais em cupuaçuzeiro (Theobroma grandiflorum) cultivado em solução nutritiva. Scientia Agrícola 51(3):407414.

SANTI A., CAMARGOS S., PEREIRA W., SCARAMUZZA J. 2006. The macronutrients deficiency in sorghum. Ciênc. Agrotec. 30(2):228-233.

SARCINELLI T., RIBEIRO E., DIAS L., LYNCH L. 2004. Sintomas de deficiência nutricional em mudas de Acacia holosericea em resposta à omissão de macronutrientes. Revista Árvore 25(2):173-181.

TAIZ L., ZEIGER E. 1998. Plant physiology. Sinauer Associates, Sunderland, Massachusetts, 792 p.

WALLAU R., BORGES A., REZENDE D., CAMARGOS S. 2008. Sintomas de deficiências nutricionais em mudas de mogno em solução nutritiva. Cerne 14(4):304-310.

ZAS R., SERRADA R. 2003. Foliar nutrient status and nutritional relationships of young Pinus radiata D. on plantations in northwest Spain. Forest Ecology and Management 174:167-176. 
